\title{
¿Por qué Leer Bien es Importante? Asociación del Dominio Lector con Otros Aprendizajes
}

\section{Why Reading Well is so Important? Association Between Reading Mastery and Other Learning Processes}

\author{
Teresa Marchant \\ Graciela Lucchini \\ Blanca Cuadrado \\ Fundación Educacional Arauco
}

\begin{abstract}
Fundación Educacional Arauco ${ }^{1}$ (Fundar), a partir de los datos obtenidos en las evaluaciones de sus distintos programas e investigaciones, realizó un estudio en una muestra de 1782 alumnos de $2^{\circ}$ a $8^{\circ}$ básico de distintas regiones de Chile, evaluados entre los años 2001 y 2005, con el objetivo de analizar la asociación entre el Dominio Lector y el desempeño en otras áreas de aprendizaje. Los resultados de este estudio muestran una significativa asociación entre la Calidad de Lectura Oral de los alumnos y el dominio de distintas habilidades como comprensión lectora, vocabulario y redacción, el mejor uso de contenidos de lenguaje, matemática y comprensión del medio, además del desarrollo de la autoestima. Interesa compartir estos resultados para destacar la importancia de desarrollar tempranamente, junto con otras habilidades, el proceso lector de los alumnos y fortalecerlo hasta lograr su total dominio.
\end{abstract}

Palabras Clave: dominio lector, lectura, comprensión, aprendizajes, autoestima.

\begin{abstract}
"Fundación Educacional Arauco (Fundar)" using data from the evaluation of programs and research, from different counties of Chile during the years 2001 to 2005, conducted a study of 1782 students from first to eight grade, to analize the association between Level of Reading Mastery and their performance in other areas of learning. The results of this study show a significant association between the students level of "Oral Reading Quality" and their performance in different skills such as reading comprehension, use of vocabulary and writing, the management of contents in Language, Mathematics, History and Science, as well as with their level self-esteem. It is interesting to share these results in order to enhance the importance of early development of the student's reading process, together with other skills, and to strengthen it until they reach full mastery.
\end{abstract}

Keywords: level of reading mastery, reading comprehension, learning, self-esteem.

Desde 1990, aumentar la cobertura y especialmente mejorar la calidad y equidad de la educación, ha sido un tema relevante en las políticas públicas del país. Sin embargo, a pesar de los diversos esfuerzos realizados, recursos involucrados y de los avances logrados en distintos ámbitos, aún queda mucho por hacer para superar la lentitud de los progresos y la inequidad persistente.

La incorporación de la investigación educacional seria, que facilite la toma de decisiones de las mejores prácticas, y de instrumentos y estándares para monitorear los progresos de los alumnos, es muy importante en los países que presentan mejores resultados.

Teresa Marchant, Fundación Educacional Arauco, Chile. Graciela Lucchini, Fundación Educacional Arauco, Chile. Blanca Cuadrado, Fundación Educacional Arauco, Chile. La correspondencia relativa al artículo deberá ser dirigida a Teresa Marchant, Fundación Educacional Arauco, Av. Santa María 2120, Providencia, Santiago-Chile. E-mail: fundacion@ arauco.cl
En este contexto siguen siendo fundamentales y válidas todas las acciones de apoyo que han emprendido o puedan emprender las instituciones que se interesan por el desarrollo, investigación y acciones dirigidas a la educación.

\section{Importancia de la Lectura y de Otras Destrezas}

Marchant, Recart, Cuadrado y Sanhueza (2004) plantean que el desarrollo de las funciones cognitivas (lenguaje oral, memoria, atención, nociones de espacio y tiempo) y del lenguaje escrito es tan importante, que la mayoría de los programas dirigidos tan-

\footnotetext{
Fundación Educacional Arauco es una institución privada sin fines de lucro, dependiente de la empresa Celulosa Arauco y Constitución S.A., que ha hecho aportes tendientes al mejoramiento de la calidad de la educación en comunas de la VII, VIII y X Regiones. Desde 1991 a la fecha, habrá beneficiado a 3.400 profesores o agentes educativos de 440 escuelas o centros de atención, en 21 comunas, favoreciendo indirectamente a más de 68.000 niños.
} 
to a niños, adolescentes, como a adultos deprivados culturalmente, se centran en el desarrollo de estas funciones y no en la entrega de contenidos. En el mundo de hoy, en que los conocimientos adquiridos rápidamente pasan a ser obsoletos, lo fundamental parece ser disponer de herramientas adecuadas para enfrentar nuevos contenidos, nuevas realidades.

La importancia del lenguaje se valora cada vez más en la pedagogía moderna. El lenguaje no sólo define al ser humano sino también a las relaciones fundamentales que establecemos con la realidad interior y con el mundo que nos rodea (Alliende \& Condemarín, 1982). La estimulación de las habilidades verbales constituye la base del desarrollo del pensamiento del niño y de la calidad de sus futuros aprendizajes.

En sus investigaciones, algunos autores (Bravo, 2003; Bravo, Villalón \& Orellana, 2002) plantean que una parte importante del éxito en el aprendizaje de la lectura depende del desarrollo cognitivo y psicolingüístico que se adquiere en los años anteriores a primero básico, corroborando lo planteado en diversos estudios internacionales de seguimiento de niños. Lograr este desarrollo se plantea como el objetivo de la educación en los primeros años de vida.

El discurso oral constituye el fundamento del discurso escrito, por lo tanto, si no se desarrolla el vocabulario y las capacidades de comprensión oral y auditiva del niño, tampoco se podrán desarrollar sus capacidades de lectura. Si las aptitudes de un niño para leer y escuchar son pobres por el hecho de haber crecido en un ambiente lingüístico limitado, deberán hacerse esfuerzos tanto para fomentar la comprensión oral y auditiva como la mecánica de la lectura (Vellutino \& Scanlon, 2001; Whitehurst \& Lonigan, 1998 citados en Marchant et al., 2004).

Pardo (2006), experto en lenguaje y fonoaudiología, señala con preocupación que las escuelas vulnerables deben tener presente la problemática del vocabulario reducido y su efecto sobre el aprendizaje inicial de la lecto-escritura. Según el autor, citando a Sawyer y Butler (1991), la competencia para leer la construimos sobre la base de la lengua, la que implica: fonología o estructura de los sonidos, la sintaxis u ordenamiento secuencial de las palabras en frases y oraciones, y la semántica o sistema de significados, factores dependientes a su vez de la memoria de corto y largo plazo.

Una vez que el alumno desarrolla un buen lenguaje oral auditivo y logra fluidez para decodificar, comienza a verificarse una influencia inversa en los cursos superiores de la enseñanza primaria. La lectu- ra se va transformando en una fuente de experiencia lingüística que permite mejorar en gran medida las aptitudes para escuchar y hablar. Aprender a leer con facilidad y de manera comprensiva contribuye al aprendizaje de nuevas palabras y a la adquisición de nuevos conocimientos.

La lectura constituye una realidad privilegiada de activación y enriquecimiento de las habilidades lingüísticas, cognitivas y afectivas de los niños. Leer permite desarrollar la imaginación, activar los procesos mentales, enriquecer el vocabulario, las estructuras gramaticales y narrativas, ampliar y organizar la adquisición de la información y desarrollar la comprensión de los mensajes escritos en distintos tipos de textos. Aquellos que leen, abren su mundo, pueden recibir información y conocimientos elaborados por otros en distintas realidades.

El aprendizaje de la escritura, a su vez, permite la expresión de la creatividad, el registro de la información y el desarrollo de técnicas de estudio. También aumenta la comprensión del mundo, la retención y recuperación de contenidos, favoreciendo la ortografía e incrementando la conciencia sobre las características del lenguaje (metacognición).

El aprendizaje de la lectura y la escritura, por lo tanto, se plantea como uno de los principales retos de toda escuela. El desafío no sólo implica enseñar la mecánica de ambos procesos hasta su total dominio, sino hacerlo tempranamente y desarrollar el "gusto por leer".

En lectura es importante tanto el desarrollo de la lectura oral como el de la lectura silenciosa. Al planear las lecciones de lectura, hay que dar gran importancia a leer en voz alta, considerando al maestro y el alumno, lo que proporciona múltiples beneficios. En primer lugar, el maestro puede servir de ejemplo de buena lectura en voz alta, al leer correctamente, con fluidez, inflexión, calidad vocal y expresión. El alumno quizás no lee tan bien como podría, porque no oye a nadie leer mejor que él. La cercanía emocional puede también ser un beneficio adicional. Ver al profesor emocionarse por los libros o expresar temor o gran alivio por causa de los eventos en una historia resuena afectivamente en el niño y lo puede ayudar a desarrollar un mayor vínculo de entendimiento. Los niños a quienes se les lee, suelen desempeñarse mejor en las pruebas de vocabulario y comprensión (Connie, 2004).

Paredes y Sassoon (2005) relacionan también fuertemente la lectura, la escritura y el desarrollo afectivo y señalan que:

Es preocupante que en la actualidad, todavía en muchos casos, se considere la escuela primaria 
sólo como un centro de aprendizaje y se pierda de vista que este espacio puede ofrecer al alumno una formación integral que comprenda tanto el desarrollo intelectual como el aspecto afectivo. (p.1)

Por otra parte, es clara y reconocida la relación entre buen rendimiento y autoestima alta. Los niños con buen rendimiento suelen confiar en sus capacidades y sentirse autoeficaces y valiosos (Haeussler \& Milicic, 1995).

La lectura y la escritura pueden ser un excelente medio para desarrollar la autoestima en el niño, como afirma Rey (1993 citado en Paredes \& Sassoon, 2005):

Con la práctica de la lectura los niños no sólo se divierten y desarrollan su vocabulario, conocimientos e imaginación; no sólo se aproximan y aprenden el uso de las estructuras de la lengua; aprenden a comunicarse con su ser interior, con esas partes desconocidas o rechazadas en él mismo; desde allí, al hablar con su interior, inician la comunicación profunda con el otro, y con los otros. Además, la lectura y la escritura proporcionan un ambiente afectivo, tanto por las historias que se pueden hallar en los libros como por la relación estrecha que se entabla con los padres de familia. La voz de la mamá y del papá al leer un cuento, cantar una canción, decir un poema o un trabalenguas, proporciona al niño la seguridad que propiciará su desarrollo cognitivo y emocional. (p. 1)

Finalmente, de acuerdo al documento "Hacia las Sociedades del Conocimiento" (UNESCO, 2005) se plantea que en el mundo contemporáneo los conocimientos básicos, a pesar de estar viviendo en una sociedad globalizada, tecnologizada y con una expansión exponencial de los conocimientos, tanto la escritura como la contabilidad son elementos omnipresentes e indispensables para la vida cotidiana y el ejercicio de la ciudadanía. El dominio lector, la escritura y el cálculo elemental, siguen siendo objetivos primordiales para dominar los procesos que rigen el aprender a aprender y poder seguir un itinerario educativo en estructuras formales y no formales. Buscar, jerarquizar y organizar la información omnipresente es el objetivo de la information literacy, $\sin$ la cual no es posible estar en las sociedades del conocimiento continuo.

\section{Fundación Educacional Arauco y la Evaluación de la Lectura}

En el marco del desarrollo y evaluación de sus programas de perfeccionamiento docente, un ámbito de trabajo importante para Fundar ha sido la evaluación del Dominio Lector como destreza básica y fundamental en el aprendizaje. Fruto de años de experiencia e investigación en el tema, Fundar ha desarrollado un set de instrumentos válidos y confiables para la evaluación de esta destreza en alumnos de $2^{\circ}$ a $8^{\circ}$ básico, como un screening rápido y simple denominado "Pruebas de Dominio Lector Fundar para alumnos de enseñanza básica" (Marchant et al., 2004).

Al evaluar Dominio Lector se consideran dos aspectos: calidad y velocidad de lectura oral. La calidad de la lectura implica determinar el grado de fluidez con que se lee. Para esto, Fundar propone fijarse en las pausas que hace el niño al leer en voz alta: después de cada sílaba, de cada palabra, de grupos de palabras o es capaz de respetar las unidades de sentido. Las siguientes 5 categorías permiten caracterizar la calidad de la lectura oral predominante: No Lector, Lectura Silábica, Lectura Palabra a Palabra, Lectura Unidades Cortas, Lectura Fluida.

El segundo aspecto importante de evaluar en Dominio Lector es la velocidad de la lectura oral. Fundar propone cronometrar el tiempo exacto que demora cada niño, en leer el texto de principio a fin y calcular el número de palabras que lee en un minuto.

La metodología de evaluación propuesta es simple y rápida (menos de $5 \mathrm{~min}$. por alumno), de tipo screening, en la que se enfrenta a cada uno de los alumnos de un curso a que lean un mismo texto completo y se evalúa cuán bien pueden leerlo y cuánto demoran en hacerlo. Los alumnos se ven enfrentados a un texto que tiene una misma longitud (número de palabras), un mismo nivel de dificultad y complejidad, un mismo tipo de letra, una cierta extensión y un sentido completo y adecuado al nivel de escolaridad del niño.

Las Pruebas de Dominio Lector Fundar tienen criterios de logro para la interpretación de resultados tanto en calidad de lectura oral como en velocidad, que establecen qué es lo esperado al inicio de cada curso de Educación Básica.

La evaluación de Dominio Lector permite precisar si hay o no un problema de decodificación. Sirve como parámetro diagnóstico en cuanto al nivel lector de los alumnos, los cursos y la escuela, y así poder establecer prioridades y metas de trabajo sobre una base real.

Para dar cuenta del nivel de comprensión lectora de un alumno, es necesario descartar que las dificultades para rendir en una prueba se deban a dificultades para decodificar el texto. Si bien la importancia de la lectura radica en lograr un buen 
nivel de comprensión, los alumnos deben contar tanto con herramientas para lograr un buen análisis y comprensión del texto como con un buen nivel de decodificación de éste, que les permita centrar su atención en el contenido y hacerlo en un tiempo adecuado a las exigencias.

\section{Método}

Fundar ha realizado diversos estudios que han permitido validar los instrumentos desarrollados, contar con criterios de logro basados en parámetros referenciales estudiados en Chile, y profundizar en la importancia del Dominio Lector y su relación con otras destrezas o áreas de aprendizaje como el nivel de comprensión de lectura silenciosa, el manejo de vocabulario, el nivel de redacción, el dominio de contenidos programáticos en lenguaje, matemáticas y comprensión del medio y la autoestima.

Ha resultado de gran interés conocer el grado de relación de los resultados alcanzados por los alumnos en lectura oral con los obtenidos en pruebas que miden otras habilidades por razones de investigación y prácticas. Resulta importante saber si la evaluación de Dominio Lector puede dar señales de lo que está sucediendo, con un adecuado nivel de certeza, con el alumno en otros aspectos del aprendizaje. Esto permitiría por una parte una economía de evaluación, y lo que es más importante tener elementos, producto de la investigación rigurosa, que convenzan a los docentes de la necesidad urgente de que los alumnos dominen su proceso lector y no permanezcan en etapas iniciales o intermedias. Las evaluaciones que han dado origen a estos estudios se han realizado en los distintos programas de capacitación docente desarrollados por Fundar en diversas comunas del país. En este trabajo, se presentan las investigaciones realizadas entre 2001 y 2005, en la Región Metropolitana (2001), en la VII Región (Constitución, 2004) y en la VIII Región (Arauco, 2002-2005; Tirúa, 2003). Se analizaron los resultados de 1782 alumnos de distintos cursos de enseñanza básica, evaluados al inicio y/o término de los respectivos programas (ver Tabla 1):

Para evaluar las distintas destrezas y habilidades se utilizaron principalmente pruebas desarrolladas y validadas en Chile, pruebas estandarizadas en Estados Unidos y también se consideraron los resultados de las pruebas nacionales (SIMCE).

Las pruebas fueron administradas por evaluadores debidamente capacitados por el equipo profesional de Fundar (psicólogos, profesores y alumnos de los últimos años de psi- cología o pedagogía). Se evaluó a alumnos de $2^{\circ}$ a $8^{\circ}$ básico, individualmente en Dominio Lector y colectivamente con las pruebas de comprensión lectora, vocabulario, redacción, contenidos y autoestima.

En su mayoría se evaluó la matrícula completa en los distintos niveles o cursos, en escuelas municipales de diversas comunas del sur del Chile. Sólo en la investigación realizada en Santiago se trabajó además con alumnos de escuelas particular pagadas y particular subvencionadas.

Los resultados que se presentan a continuación buscan contrastar las siguientes hipótesis de trabajo:

1. La evaluación de la calidad de lectura, descrita como la capacidad para decodificar desde letras, sílabas, palabras, párrafos cortos hasta párrafos con sentido, se relaciona estadísticamente con otras destrezas tales como vocabulario, comprensión, redacción, rendimiento en pruebas de conocimiento en diversas áreas y con autoestima.

2. Existe asociación entre calidad y velocidad de lectura oral, dos aspectos que conforman el concepto de Dominio Lector.

Considerando que las Pruebas de Dominio Lector entregan resultados en términos de categorías de lectura, y las otras pruebas estudiadas entregan resultados en escala de puntaje, para el análisis de los resultados se utilizó el test estadístico múltiple de comparación de pares de promedios, Sidak. Este test garantiza la comparación de todos los pares de promedios a un nivel de significancia fijo alfa. En este caso, se buscó establecer si los promedios de resultados obtenidos en distintas destrezas por grupos de alumnos con distinto nivel de lectura resultaban estadísticamente significativas. Para la presentación de los resultados, en el Test Sidak se entrega una letra asociada a cada promedio. Si dos promedios no tienen "ninguna" letra en común, diremos que existe evidencia estadística para señalar que los promedios son distintos.

\section{Resultados}

\section{Asociación Calidad de Lectura Oral-Otras Habilidades}

Calidad de lectura oral y comprensión de lectura silenciosa. La asociación Calidad de Lectura Oral-Comprensión de Lectura Silenciosa ha sido estudiada en comunas de tres regiones y tres cursos de Educación Básica (ver Tabla 2).

En cada muestra se evaluó calidad de lectura oral con las Pruebas de Dominio Lector Fundar y comprensión de lectura silenciosa con la Prueba

\section{Tabla 1}

Muestras y cursos en que fue estudiada la asociación entre Calidad de Lectura Oral y otros aprendizajes

\begin{tabular}{llc}
\hline Lugar y año & Cursos & Muestra alumnos $(n)$ \\
\hline Santiago, 2001 & $3^{\circ}, 6^{\circ}, 8^{\circ}$ & 394 \\
Arauco, 2002 & $4^{\circ}$ & 87 \\
Tirúa, 2003 & $2^{\circ}, 3^{\circ}, 6^{\circ}, 8^{\circ}$ & 656 \\
Constitución, 2004 & $3^{\circ}, 6^{\circ}, 8^{\circ}$ & 543 \\
Arauco, 2005 & $4^{\circ}$ & 102 \\
\hline
\end{tabular}


Tabla 2

Muestra de alumnos evaluados en Calidad de Lectura Oral y Comprensión de Lectura

\begin{tabular}{cccc}
\hline & SANTIAGO & TIRÚA & CONSTITUCIÓN 2004 \\
Curso & 2001 & 2003 & $n$ \\
& $n$ & $n$ & 155 \\
$3^{\circ}$ & 133 & 124 & 206 \\
$6^{\circ}$ & 131 & 173 & 182 \\
$8^{\circ}$ & 130 & 177 & \\
\hline
\end{tabular}

Tabla 3

Asociación entre Calidad de Lectura y Comprensión de Lectura. Santiago, 2001

\begin{tabular}{|c|c|c|c|c|c|c|c|c|c|}
\hline \multirow{3}{*}{$\begin{array}{l}\text { CURSO } \\
\text { CATEGORÍAS } \\
\text { LECTURA }\end{array}$} & \multicolumn{9}{|c|}{ COMPRENSIÓN } \\
\hline & \multicolumn{3}{|c|}{$3^{\circ}(n=133)$} & \multicolumn{3}{|c|}{$6^{\circ}(n=131)$} & \multicolumn{3}{|c|}{$8^{\circ}(n=130)$} \\
\hline & $n$ & $\begin{array}{c}\bar{X} \\
\text { percentil }\end{array}$ & $\begin{array}{l}\text { Test } \\
\text { Sidak }\end{array}$ & $n$ & $\begin{array}{c}\bar{X} \\
\text { percentil }\end{array}$ & $\begin{array}{l}\text { Test } \\
\text { Sidak }\end{array}$ & $n$ & $\begin{array}{c}\bar{X} \\
\text { percentil }\end{array}$ & $\begin{array}{r}\text { Test } \\
\text { Sidak }\end{array}$ \\
\hline No Lector & 9 & 11.3 & $\mathrm{C}$ & & & & & & \\
\hline Silábica & 11 & 14.5 & $\mathrm{C}$ & & & & & & \\
\hline $\begin{array}{l}\text { Palabra a } \\
\text { Palabra }\end{array}$ & 43 & 34.6 & B & 8 & 33.1 & $\mathrm{C}$ & 2 & 17.0 & B \\
\hline $\begin{array}{l}\text { Unidades } \\
\text { Cortas }\end{array}$ & 48 & 50.3 & A & 59 & 60.0 & B & 49 & 53.0 & B \\
\hline Fluida & 22 & 54.3 & A & 64 & 69.0 & A & 79 & 66.9 & A \\
\hline
\end{tabular}

*En el Test Sidak, letras distintas indican diferencias estadísticamente significativas (valor $p<0.05)$ entre los grupos, en cada curso.

de Lectura y Lenguaje Escrito-PLLE, desarrollada por autores norteamericanos (Hammill, Larsen, Wiederholt \& Fountain-Chambers, 1982). De esta última, se consideraron tres subpruebas: Lectura de Párrafos que evalúa comprensión de lectura silenciosa, Vocabulario de Lectura que evalúa dominio de vocabulario y Composición que evalúa redacción. Cada subprueba tiene normas por edad y entrega resultados en puntaje estándar y percentiles.

En la Tabla 3, se presenta el estudio de la relación entre calidad de lectura oral y comprensión de lectura silenciosa, en una muestra de 394 alumnos de $3^{\circ}, 6^{\circ}$ y $8^{\circ}$ Básico de establecimientos de distinta dependencia y nivel socioeconómico de Santiago (2001).

En cada curso se aprecia una relación directa entre calidad lectora y comprensión de lectura silenciosa. A mejor categoría de lectura hay un mayor puntaje en comprensión expresado en promedio de percentiles.

Para conocer si existía una relación estadísticamente significativa entre los resultados obtenidos en el nivel de comprensión lectora de acuerdo a la calidad de lectura de los alumnos se realizó un análisis al interior de cada curso. La Tabla 3 muestra el sentido de la asociación para cada uno de los cursos en la muestra de Santiago 2001. Puede verse una tendencia positiva en el promedio de comprensión lectora de cada curso, conforme los alumnos presentan un mayor nivel de Dominio Lector, observándose diferencias significativas en el rendimiento entre los niños que presentan distinta calidad de lectura.

En $3^{\circ}$ Básico, los niños con menor Dominio Lector (No Lectores, Lectura Silábica) alcanzan un rendimiento, en comprensión lectora, significativamente inferior al de los alumnos con Lectura Palabra a Palabra. A su vez, los alumnos que leen Palabra a Palabra presentan un rendimiento significativamente inferior al alcanzado en comprensión por los niños que presentan mayor Dominio Lector (L. Unidades Cortas, L. Fluida). En $6^{\circ}$ Básico, se aprecia que el rendimiento en comprensión también presenta diferencias, estadísticamente significativas, para cada una de las categorías de lectura alcanzadas. En $8^{\circ}$ Bá- 
Tabla 4

Asociación entre Calidad de Lectura Oral y Comprensión de Lectura Silenciosa. Constitución, 2004

\begin{tabular}{|c|c|c|c|c|c|c|c|c|c|}
\hline \multirow{3}{*}{$\begin{array}{l}\text { CURSO } \\
\text { CATEGORÍAS } \\
\text { LECTURA }\end{array}$} & \multicolumn{9}{|c|}{ COMPRENSIÓN } \\
\hline & & $3^{\circ}$ & & & $6^{\circ}$ & & & & \\
\hline & $n$ & $\begin{array}{c}\bar{X} \\
\text { percentil }\end{array}$ & $\begin{array}{c}\text { Test } \\
\text { Sidak }\end{array}$ & $n$ & $\begin{array}{c}\bar{X} \\
\text { percentil }\end{array}$ & $\begin{array}{c}\text { Test } \\
\text { Sidak }\end{array}$ & $n$ & $\begin{array}{c}\bar{X} \\
\text { percentil }\end{array}$ & $\begin{array}{c}\text { Test } \\
\text { Sidak }\end{array}$ \\
\hline No Lector & 1 & $37.0^{*}$ & & & & & & & \\
\hline Silábica & 47 & 17.3 & $\mathrm{C}$ & 3 & 34.7 & & & & \\
\hline $\begin{array}{l}\text { Palabra a } \\
\text { Palabra }\end{array}$ & 54 & 32.8 & B & 26 & 33.7 & $\mathrm{C}$ & 8 & 39.9 & B \\
\hline $\begin{array}{l}\text { Unidades } \\
\text { Cortas }\end{array}$ & 41 & 49.9 & A & 106 & 50.6 & B & 97 & 44.2 & B \\
\hline Fluida & 12 & 53.4 & A & 71 & 60.5 & A & 77 & 60.8 & A \\
\hline TOTAL & 155 & 34.3 & $<0.001$ & 206 & 51.6 & $<0.001$ & 182 & 51.0 & $<0.001$ \\
\hline
\end{tabular}

*Corresponde a la respuesta aleatoria de un alumno no lector.

Tabla 5

Criterios de logro en Calidad de Lectura Oral por curso

\begin{tabular}{|c|c|c|}
\hline CURSO & $\begin{array}{c}\text { NIVEL DE LECTURA } \\
\text { ESPERADO }\end{array}$ & $\begin{array}{l}\text { NIVEL DE LECTURA } \\
\text { BAJO LO ESPERADO }\end{array}$ \\
\hline $3^{\circ}$ & $\begin{array}{l}\text { Unidades Cortas } \\
\text { Fluida }\end{array}$ & $\begin{array}{l}\text { No Lector } \\
\text { Silábica } \\
\text { Palabra a Palabra }\end{array}$ \\
\hline $6^{\circ}$ & Fluida & $\begin{array}{l}\text { No Lector } \\
\text { Silábica } \\
\text { Palabra a Palabra } \\
\text { Unidades Cortas }\end{array}$ \\
\hline $8^{\circ}$ & Fluida & $\begin{array}{l}\text { No Lector } \\
\text { Silábica } \\
\text { Palabra a Palabra } \\
\text { Unidades Cortas }\end{array}$ \\
\hline
\end{tabular}

sico se observa que los alumnos que leen fluidamente presentan un rendimiento estadísticamente superior al de los niños con menor Dominio Lector.

Esta asociación positiva y las diferencias estadísticamente significativas entre categorías no sólo se dieron en esta investigación sino también en las realizadas en la comuna de Tirúa (VIII Región), en el año 2003 y en la comuna de Constitución (VII región), en el año 2004. La Tabla 4 muestra esta asociación en la muestra de Constitución en que se evaluaron 543 alumnos de educación básica de escuelas municipales.

En otra mirada de los datos, se estudió el comportamiento en comprensión lectora, de los niños que obtuvieron un nivel lector esperado para su nivel de escolaridad en comparación a los niños que obtuvieron un nivel menor al esperado. La Tabla 5 muestra la categorización utilizada para cada curso (Marchant, et al., 2004).

Al agrupar los resultados alcanzados en comprensión lectora, de acuerdo a los niveles esperados y no esperados de calidad de lectura, se observó la siguiente asociación en la muestra de Tirúa, el año 2003 (ver Tabla 6) 2 :

En todos los cursos existe una diferencia estadísticamente significativa en el nivel de compren-

2 Los resultados por categoría de lectura están disponibles en Fundar. 
Tabla 6

Asociación entre Calidad de Lectura Oral y Comprensión de Lectura Silenciosa por criterio de logro. Tirúa, 2003

\section{COMPRENSIÓN}

\begin{tabular}{|c|c|c|c|c|c|c|c|c|c|}
\hline CURSO & & $3^{\circ}$ & & & $6^{\circ}$ & & & $8^{\circ}$ & \\
\hline $\begin{array}{l}\text { NIVEL DE } \\
\text { LOGRO } \\
\text { DE LECTURA }\end{array}$ & $n$ & $\begin{array}{c}\bar{X} \\
\text { percentil }\end{array}$ & $\begin{array}{l}\text { Test } \\
\text { Sidak }\end{array}$ & $n$ & $\begin{array}{c}\bar{X} \\
\text { percentil }\end{array}$ & $\begin{array}{c}\text { Test } \\
\text { Sidak }\end{array}$ & $n$ & $\begin{array}{c}\bar{X} \\
\text { percentil }\end{array}$ & $\begin{array}{c}\text { Test } \\
\text { Sidak }\end{array}$ \\
\hline Bajo lo esperado & 66 & 15.4 & B & 89 & 23.7 & B & 59 & 28.5 & B \\
\hline Esperado & 58 & 36.1 & A & 84 & 47.4 & A & 118 & 45.7 & A \\
\hline TOTAL & 124 & 25.1 & $<0.001$ & 173 & 35.2 & $<0.001$ & 177 & 39.9 & $<0.001$ \\
\hline
\end{tabular}

Tabla 7

Asociación entre Calidad de Lectura Oral y Vocabulario. Constitución, 2004

\begin{tabular}{|c|c|c|c|c|c|c|}
\hline \multicolumn{7}{|c|}{ VOCABULARIO } \\
\hline CURSO & & $3^{\circ}$ & & & $6^{\circ}$ & \\
\hline $\begin{array}{l}\text { CATEGORÍAS } \\
\text { LECTURA }\end{array}$ & $n$ & $\begin{array}{c}\bar{X} \\
\text { percentil }\end{array}$ & $\begin{array}{l}\text { Test } \\
\text { Sidak }\end{array}$ & $n$ & $\begin{array}{c}\bar{X} \\
\text { percentil }\end{array}$ & $\begin{array}{l}\text { Test } \\
\text { Sidak }\end{array}$ \\
\hline No Lector & 1 & & & & & \\
\hline Silábica & 47 & 22.7 & $\mathrm{C}$ & 3 & 25.7 & \\
\hline Palabra a Palabra & 54 & 34.5 & $\mathrm{BC}$ & 26 & 24.2 & $\mathrm{C}$ \\
\hline Unidades Cortas & 41 & 40.5 & B & 106 & 42.1 & B \\
\hline Fluida & 12 & 60.3 & A & 71 & 55.5 & A \\
\hline TOTAL & 155 & 34.3 & $<0.001$ & 206 & 44.2 & $<0.001$ \\
\hline
\end{tabular}

sión, entre aquellos niños cuya calidad de lectura oral corresponde al nivel esperado para su curso y aquellos que presentan una calidad de lectura menor que la esperada.

De esta forma, se puede señalar que existe una asociación positiva entre la calidad de lectura oral, evaluada a través de las Pruebas de Dominio Lector Fundar, y el nivel de comprensión de lectura silenciosa evaluado a través de la subprueba Lectura de Párrafos de la prueba PLLE. Las relaciones observadas entre calidad de lectura oral y comprensión de lectura silenciosa, en las evaluaciones realizadas tanto en Santiago como en las comunas de Tirúa y Constitución, confirman la asociación teórica establecida entre ambos aspectos, toda vez que se ha afirmado que la comprensión es posible cuando hay un mínimo nivel lector. Estos análisis corroboran que los mejores resultados en comprensión se observan cuando se ha alcanzado fluidez en la lectura oral.
Calidad de lectura oral y dominio de vocabulario. La asociación Calidad de Lectura Oral-Dominio de Vocabulario fue estudiada en dos muestras y varios cursos de educación básica.

En cada muestra se evaluó calidad de lectura oral con las Pruebas de Dominio Lector Fundar y dominio de Vocabulario con la PLLE (Subprueba: Vocabulario de Lectura).

En la Tabla 7, se presenta el estudio de la relación entre calidad de lectura oral y dominio de vocabulario para los resultados de 361 alumnos de $3^{\circ}$ y $6^{\circ}$ Básico de escuelas municipales de la Comuna de Constitución, VII Región.

Puede observarse en cada curso una relación directa entre calidad lectora y dominio de vocabulario.

Se observan diferencias significativas al interior de los cursos entre los niños que presentan distinta calidad de lectura. En cada curso, los alumnos rinden significativamente mejor en vocabulario conforme mejoran su nivel lector. 
Tabla 8

Asociación entre Calidad de Lectura Oral y Vocabulario según criterio de logro. Tirúa, 2003

\begin{tabular}{|c|c|c|c|c|c|c|c|c|c|}
\hline \multirow{2}{*}{$\begin{array}{l}\text { CURSO } \\
\text { NIVEL DE } \\
\text { LOGRO } \\
\text { DE LECTURA }\end{array}$} & \multicolumn{9}{|c|}{ VOCABULARIO } \\
\hline & $n$ & $\begin{array}{c}\bar{X} \\
\text { percentil }\end{array}$ & $\begin{array}{c}\text { Test } \\
\text { Sidak }\end{array}$ & $n$ & $\begin{array}{c}\bar{X} \\
\text { percentil }\end{array}$ & $\begin{array}{c}\text { Test } \\
\text { Sidak }\end{array}$ & $n$ & $\begin{array}{c}\bar{X} \\
\text { percentil }\end{array}$ & $\begin{array}{l}\text { Test } \\
\text { Sidak }\end{array}$ \\
\hline Bajo lo esperado & 67 & 15.4 & B & 88 & 25.9 & B & 59 & 32.9 & B \\
\hline Esperado & 58 & 34.0 & A & 84 & 44.0 & A & 118 & 46.6 & A \\
\hline TOTAL & 125 & 24.0 & $<0.001$ & 172 & 34.8 & $<0.001$ & 177 & 42.1 & 0.001 \\
\hline
\end{tabular}

Tabla 9

Asociación entre Calidad de Lectura Oral y Redacción. Tirúa, 2003

\begin{tabular}{|c|c|c|c|c|c|c|c|c|c|}
\hline \multirow{3}{*}{$\begin{array}{l}\text { CURSO } \\
\text { CATEGORÍAS } \\
\text { LECTURA }\end{array}$} & \multicolumn{9}{|c|}{ REDACCIÓN } \\
\hline & & $3^{\circ}$ & & & $6^{\circ}$ & & & $8^{\circ}$ & \\
\hline & $n$ & $\begin{array}{c}\bar{X} \\
\text { percentil }\end{array}$ & $\begin{array}{c}\text { Test } \\
\text { Sidak }\end{array}$ & $n$ & $\begin{array}{c}\bar{X} \\
\text { percentil }\end{array}$ & $\begin{array}{c}\text { Test } \\
\text { Sidak }\end{array}$ & $n$ & $\begin{array}{c}\bar{X} \\
\text { percentil }\end{array}$ & $\begin{array}{l}\text { Test } \\
\text { Sidak }\end{array}$ \\
\hline No Lector & 8 & 1.1 & $\mathrm{C}$ & & & & & & \\
\hline Lectura Silábica & 13 & 22.2 & $\mathrm{BC}$ & & & & & & \\
\hline Palabra a Palabra & 22 & 43.1 & $\mathrm{AB}$ & 17 & 30.8 & B & & & \\
\hline Unidades Cortas & 34 & 53.9 & A & 45 & 49.8 & $\mathrm{AB}$ & 40 & 39.8 & B \\
\hline Fluida & 9 & 72.6 & A & 48 & 60.4 & A & 73 & 54.4 & A \\
\hline TOTAL & 86 & 43.4 & $<0.001$ & 110 & 51.5 & 0.001 & 113 & 49.1 & 0.013 \\
\hline
\end{tabular}

En otra mirada de los datos de la muestra de la comuna de Tirúa (VIII Región), en que se evaluó a 473 alumnos de $3^{\circ}, 6^{\circ}$ y $8^{\circ}$ Básico, se observó el comportamiento en vocabulario de los niños que obtuvieron un nivel lector esperado para su nivel de escolaridad en comparación a los niños que obtuvieron un nivel menor al esperado ${ }^{3}$. Para hacerlo, se consideró la misma categorización detallada en el estudio de asociación entre calidad de lectura oral y comprensión lectora (ver Tabla 5). En la Tabla 8 se presenta la asociación observada.

Se aprecia una diferencia estadísticamente significativa en el nivel de vocabulario entre aquellos niños cuya calidad de lectura oral corresponde al nivel esperado para su curso y aquellos que presentan una calidad de lectura menor que la esperada.

Como se puede observar, existe una asociación positiva entre la calidad de lectura oral, evaluada a

3 Los resultados por categoría de lectura están disponibles en Fundar. través de las Pruebas de Dominio Lector Fundar, y el nivel de vocabulario evaluado a través de la subprueba Vocabulario de Lectura (PLLE).

Calidad de lectura oral y redacción. La asociación Calidad de Lectura Oral-Redacción fue estudiada en dos muestras: una muestra de 309 alumnos de $3^{\circ}, 6^{\circ}$ y $8^{\circ}$ Básico de escuelas municipales de la comuna de Tirúa y otra de 361 alumnos de $3^{\circ}$ y $6^{\circ}$ Básico de alumnos de escuelas municipales en la comuna de Constitución.

En ambos estudios se evaluó calidad de lectura oral con las Pruebas de Dominio Lector Fundar y Redacción con la PLLE (Subprueba: Composición).

En la Tabla 9 se presentan los resultados obtenidos en la comuna de Tirúa.

Puede observarse una tendencia positiva en el promedio de redacción de cada uno de los cursos, conforme los alumnos presentan un mayor nivel de Dominio Lector. En cada uno de los cursos los alumnos rinden significativamente mejor en redacción conforme mejoran su nivel lector. 
Tabla 10

Promedio de resultados SIMCE 2002 (4ª́sico) por subsector de acuerdo a Calidad de Lectura Oral, $n=85$. Comuna de la provincia de Arauco, 2002

\begin{tabular}{|c|c|c|c|c|c|c|}
\hline \multirow{3}{*}{$\begin{array}{l}\text { CATEGORÍAS } \\
\text { LECTURA }\end{array}$} & \multicolumn{6}{|c|}{ SIMCE } \\
\hline & \multicolumn{2}{|c|}{ LENGUAJE } & \multicolumn{2}{|c|}{ MATEMÁTICA } & \multicolumn{2}{|c|}{ COMPRENSIÓN DEL MEDIO } \\
\hline & $\bar{X}$ & Desv. Estándar & $\bar{X}$ & Desv. Estándar & $\bar{X}$ & Desv. Estándar \\
\hline \multicolumn{7}{|l|}{ No lector } \\
\hline Silábica & 182 & 39.74 & 175 & 12.27 & 181 & 55.3 \\
\hline Palabra a Palabra & 200 & 34.80 & 198 & 31.01 & 211 & 43.96 \\
\hline Unidades Cortas & 231 & 37.24 & 223 & 42.59 & 224 & 43.06 \\
\hline Fluida & 291 & 33.99 & 287 & 37.59 & 294 & 28.87 \\
\hline TOTAL & 231 & 47.61 & 224 & 48.68 & 230 & 51.29 \\
\hline
\end{tabular}

Esta asociación positiva Calidad de Lectura OralRedacción se dio también en el estudio realizado en Constitución, pero las diferencias significativas se dieron sólo en $3^{\circ}$ Básico. Los alumnos de $6^{\circ}$ incrementaron sistemáticamente sus percentiles promedio según categoría de lectura, pero las diferencias no fueron estadísticamente significativas.

Los resultados anteriores permiten señalar que existe una asociación positiva entre la calidad de lectura oral, evaluada a través de las Pruebas de Dominio Lector Fundar, y el nivel de redacción evaluado a través de la subprueba Composición (PLLE). Sería interesante hacer una nueva investigación con alumnos de $6^{\circ}$ y $8^{\circ}$ Básico.

Calidad de lectura oral y resultados SIMCE. El año 2003, Fundar solicitó al SIMCE, de la Unidad de Currículo y Evaluación del Ministerio de Educación, un estudio de asociación entre Dominio Lector y resultados SIMCE, en una muestra de 85 alumnos de $4^{\circ}$ Básico de escuelas municipales de una comuna de la Provincia de Arauco (VIII Región).

Los análisis estadísticos mostraron una relación directa entre calidad de lectura oral y resultados SIMCE en los tres subsectores: Lenguaje, Matemáticas y Comprensión del Medio (ver Tabla 10).

En este análisis se pudo apreciar que los niños con mejor calidad de lectura oral presentaron un rendimiento promedio en el SIMCE, significativamente superior $(p \leq 0.05)$ al obtenido por los niños que presentan una calidad de lectura inferior. Esto fue válido para las tres categorías superiores (Lectura Palabra a Palabra, L. Unidades Cortas, L. Fluida). En la categoría inferior (L. Silábica) no hubo suficientes casos para poder hacer este análisis.
Puede observarse (ver Tabla 10) que los alumnos de $4^{\circ}$ Básico que alcanzaron un buen nivel lector (L. Fluida) alcanzaron en promedio un resultado superior o igual a los 287 puntos en los tres subsectores, mientras los alumnos que leen Palabra a Palabra no superaron en promedio los 211 puntos.

Si se consideran los criterios de logro establecidos por el instrumento, en que se espera que los alumnos al inicio de $5^{\circ}$ Básico sean capaces al menos de leer Unidades Cortas, se observan los siguientes resultados, en la Tabla 11.

Los alumnos que alcanzan las categorías de lectura esperadas para su curso presentan un rendimiento promedio significativamente superior al observado en los alumnos de bajo nivel de logro $(p \leq 0.05)$. Esto fue válido para los tres subsectores evaluados en el SIMCE.

De esta forma, se puede señalar que en $4^{\circ}$ Básico existe una asociación positiva entre la calidad de lectura oral, evaluada a través de las Pruebas de Dominio Lector Fundar, y los resultados en los distintos subsectores, obtenidos por el SIMCE.

Calidad de Lectura oral y pruebas de contenidos Fundar. Fundar también ha elaborado pruebas para evaluar contenidos mínimos en lenguaje y matemáticas. Estas pruebas no han sido validadas, sin embargo en el análisis realizado por el Mineduc (SIMCE), las pruebas de $4^{\circ}$ Básico presentaron una buena correlación $(>0.70)$ con los resultados SIM$\mathrm{CE}$ en los diferentes subsectores. Dado esto, también se quiso estudiar la asociación de estas pruebas con el nivel de Dominio Lector.

La asociación Calidad de Lectura Oral-Pruebas de Contenidos Fundar ha sido estudiada en dos muestras de Cuarto Básico de alumnos de escuelas municipales de la comuna de Arauco. La primera in- 
Tabla 11

Promedio de Resultados Simce 2002 (4ª́sico) según criterio de logro para Calidad de Lectura Oral, $n=85$. Comuna de la provincia de Arauco, 2002

\begin{tabular}{lcccccc}
\hline & \multicolumn{9}{c}{ SIMCE } \\
NIVEL DE LOGRO & \multicolumn{2}{c}{ LENGUAJE } & \multicolumn{2}{c}{ MATEMÁTICA } & \multicolumn{2}{c}{ COMPRENSIÓN DEL MEDIO } \\
DE LECTURA & $\bar{X}$ & Desv. Estándar & $\bar{X}$ & Desv. Estándar & $\bar{X}$ & Desv. Estándar \\
\hline Bajo lo Esperado & 198 & 35.01 & 194 & 29.82 & 206 & 46.12 \\
Esperado & 247 & 44.77 & 239 & 49.41 & 242 & 49.96 \\
TOTAL & 231 & 47.61 & 224 & 48.68 & 230 & 51.29 \\
\hline
\end{tabular}

Tabla 12

Asociación entre Calidad de Lectura Oral y dominio de contenidos en Lenguaje y Matemáticas (4º́sico). Arauco, 2002

\begin{tabular}{|c|c|c|c|c|c|c|}
\hline \multirow{2}{*}{$\begin{array}{l}\text { CATEGORÍAS } \\
\text { LECTURA }\end{array}$} & \multicolumn{3}{|c|}{ LENGUAJE } & \multicolumn{3}{|c|}{ MATEMÁTICA } \\
\hline & $n$ & $\begin{array}{c}\bar{X} \\
\% \text { logro }\end{array}$ & $\begin{array}{l}\text { Test } \\
\text { Sidak }\end{array}$ & $n$ & $\begin{array}{c}\bar{X} \\
\% \text { logro }\end{array}$ & $\begin{array}{l}\text { Test } \\
\text { Sidak }\end{array}$ \\
\hline \multicolumn{7}{|l|}{ No lector } \\
\hline Silábica & 6 & 31.4 & $\mathrm{C}$ & 6 & 29.9 & $\mathrm{C}$ \\
\hline Palabra a Palabra & 24 & 42.2 & $\mathrm{C}$ & 24 & 42.0 & $\mathrm{C}$ \\
\hline Unidades Cortas & 42 & 58.0 & B & 42 & 53.7 & B \\
\hline Fluida & 15 & 78.0 & A & 15 & 77.0 & A \\
\hline TOTAL & 87 & 55.3 & $<0.001$ & 87 & 52.8 & $<0.001$ \\
\hline
\end{tabular}

vestigación se hizo con datos de 87 alumnos (2002) y la segunda, con datos de 85 alumnos (2005).

En cada caso, se evaluó calidad de lectura oral con las Pruebas de Dominio Lector Fundar y contenidos de lenguaje y matemáticas con las Pruebas Fundar. En la Tabla 12, se presenta el análisis de la muestra evaluada el año $2002^{4}$.

Puede observarse una tendencia positiva en el porcentaje de logro que alcanzan los alumnos, conforme presentan un mayor nivel de Dominio Lector. A mayor categoría de lectura se observa un mejor rendimiento tanto en lenguaje como en matemáticas. Estas diferencias fueron estadísticamente significativas.

Los resultados de estas investigaciones corroboran la información entregada en el punto anterior (Asociación Calidad de Lectura Oral-Resultados SIMCE) en relación a que existe una asociación positiva entre el nivel de calidad de lectura oral y el dominio de contenidos y habilidades que logran los alumnos tanto en los subsectores de lenguaje como de matemáticas. Tanto

\footnotetext{
${ }_{4}^{4}$ Los resultados de Arauco (2005) están disponibles en Fundar.
}

la medición SIMCE como las Pruebas de Contenidos Fundar corroboran esta hipótesis.

Calidad de lectura oral y autoestima. La asociación Calidad de Lectura Oral-Autoestima ha sido estudiada en dos muestras y varios cursos de educación básica. En cada muestra, se evaluó Calidad de Lectura Oral con las Pruebas de Dominio Lector Fundar y Autoestima con el TAE Profesor, Test de Inferencia de Autoestima (Marchant, Haeussler $\&$ Torretti, 2002). El TAE Profesor es un test de screening o tamizaje, estandarizado en nuestro país, que permite conocer la percepción que tienen los profesores del nivel de autoestima de los alumnos de enseñanza básica en relación a normas establecidas por curso y por edad.

Se analizó los resultados de 262 alumnos de $3^{\circ}$ y $6^{\circ}$ Básico de escuelas municipales de la comuna de Tirúa, VIII Región, en calidad de lectura oral y en autoestima (ver Tabla 13).

Puede observarse en ambos cursos una relación entre calidad de lectura oral y autoestima. Se observan diferencias significativas al interior de los cursos entre los niños que presentan distinta 
Tabla 13

Asociación entre Calidad de Lectura Oral y Autoestima. Tirúa, 2003

\begin{tabular}{|c|c|c|c|c|c|c|}
\hline \multirow[b]{2}{*}{$\begin{array}{l}\text { CURSO } \\
\text { CATEGORÍAS } \\
\text { LECTURA }\end{array}$} & \multicolumn{6}{|c|}{ AUTOESTIMA } \\
\hline & $n$ & $\begin{array}{c}3^{\circ} \\
\bar{X} \\
\text { Puntaje T }\end{array}$ & $\begin{array}{c}\text { Test } \\
\text { Sidak } \\
\end{array}$ & $n$ & $\begin{array}{c}6^{\circ} \\
\bar{X} \\
\text { Puntaje T }\end{array}$ & $\begin{array}{c}\text { Test } \\
\text { Sidak } \\
\end{array}$ \\
\hline No Lector & 12 & 38.3 & $\mathrm{D}$ & & & \\
\hline Silábica & 15 & 48.4 & $\mathrm{C}$ & & & \\
\hline Palabra a Palabra & 38 & 51.4 & $\mathrm{BC}$ & 17 & 50.1 & B \\
\hline Unidades Cortas & 47 & 55.7 & $\mathrm{AB}$ & 43 & 51.2 & B \\
\hline Fluida & 14 & 59.2 & A & 74 & 56.5 & A \\
\hline TOTAL & 128 & 52 & $<0.001$ & 134 & 54 & $<0.001$ \\
\hline
\end{tabular}

Tabla 14

Asociación de Calidad de Lectura Oral y Velocidad de Lectura Oral. Constitución, 2004

\begin{tabular}{|c|c|c|c|c|c|c|c|c|c|}
\hline \multirow{3}{*}{$\begin{array}{l}\text { CURSO } \\
\text { CATEGORÍAS } \\
\text { LECTURA }\end{array}$} & \multicolumn{9}{|c|}{ VELOCIDAD DE LECTURA } \\
\hline & & $3^{\circ}$ & & & $6^{\circ}$ & & & $8^{\circ}$ & \\
\hline & $n$ & $\begin{array}{c}\bar{X} \\
\mathrm{ppm}\end{array}$ & $\begin{array}{c}\text { Test } \\
\text { Sidak }\end{array}$ & $n$ & $\begin{array}{c}\bar{X} \\
\mathrm{ppm}\end{array}$ & $\begin{array}{c}\text { Test } \\
\text { Sidak }\end{array}$ & $n$ & $\begin{array}{c}\bar{X} \\
\mathrm{ppm}\end{array}$ & $\begin{array}{c}\text { Test } \\
\text { Sidak }\end{array}$ \\
\hline No Lector & 1 & & & & & & & & \\
\hline Silábica & 47 & 42.1 & $\mathrm{D}$ & 3 & 49.3 & & & & \\
\hline Palabra a Palabra & 54 & 62.3 & $\mathrm{C}$ & 26 & 55.6 & $\mathrm{C}$ & 8 & 68.4 & $\mathrm{C}$ \\
\hline Unidades Cortas & 41 & 88.5 & B & 106 & 83.2 & B & 97 & 89.7 & B \\
\hline Fluida & 12 & 111.4 & A & 71 & 110.2 & A & 77 & 121.9 & A \\
\hline TOTAL & 155 & 66.9 & $<0.001$ & 206 & 88.5 & $<0.001$ & 182 & 102.4 & $<0.001$ \\
\hline
\end{tabular}

calidad de lectura. En $3^{\circ}$ y $6^{\circ}$ Básico los alumnos presentan una autoestima significativamente más alta conforme mejoran su nivel lector. Los alumnos que leen fluidamente son los que presentan mejor autoestima.

Esta asociación positiva y las diferencias estadísticamente significativas entre categorías no sólo se dieron en la muestra de la comuna de Tirúa, sino también en el análisis realizado en la comuna de Constitución, en la VII Región, en el año 2004. En este caso, se evaluaron 361 alumnos de Educación Básica de escuelas municipales ${ }^{5}$.

Los resultados permiten dar cuenta de la asociación entre el nivel de calidad de lectura oral y el desarrollo de la autoestima. El nivel de Dominio Lector parece relacionarse no sólo con el desarrollo

\footnotetext{
5 Los resultados de Constitución están disponibles en Fundar
}

de habilidades y contenidos académicos sino también con el autoconcepto en general.

Calidad de lectura oral y velocidad de lectura oral. Dado que las Pruebas de Dominio Lector Fundar evalúan tanto calidad como velocidad de lectura oral resultaba de interés confirmar la asociación entre estas dos variables.

Para conocer si existía una relación estadísticamente significativa entre en calidad de lectura y los resultados obtenidos en velocidad de lectura, se realizó a un análisis al interior de los cursos en cada una de las muestras consideradas en esta investigación (ver Tabla 1).

En las muestras analizadas pudo observarse una relación directa entre calidad de lectura y velocidad lectora. A mejor categoría de calidad de lectura se observó un mejor promedio en velocidad lectora (expresado en palabras por minuto). 
Tabla 15

Asociación entre Calidad de Lectura Oral y otras áreas de aprendizaje (6º́sico). Constitución, 2004

\begin{tabular}{|c|c|c|c|c|c|c|c|c|c|}
\hline \multirow[b]{2}{*}{$\begin{array}{l}\text { CATEGORÍAS } \\
\text { LECTURA }\end{array}$} & \multirow[b]{2}{*}{$n$} & \multicolumn{2}{|c|}{ VLO } & \multicolumn{2}{|c|}{$\begin{array}{l}\text { Comprensión } \\
\text { PLLE }\end{array}$} & \multicolumn{2}{|c|}{$\begin{array}{l}\text { Vocabulario } \\
\text { PLLE }\end{array}$} & \multicolumn{2}{|c|}{$\begin{array}{c}\text { Autoestima } \\
\text { TAE Profesor }\end{array}$} \\
\hline & & $\begin{array}{c}\bar{X} \\
\mathrm{ppm}\end{array}$ & $\begin{array}{l}\text { Test } \\
\text { Sidak }\end{array}$ & $\begin{array}{c}\bar{X} \\
\text { Percentil }\end{array}$ & $\begin{array}{c}\text { Test } \\
\text { Sidak }\end{array}$ & $\begin{array}{c}\bar{X} \\
\text { Percentil }\end{array}$ & $\begin{array}{c}\text { Test } \\
\text { Sidak }\end{array}$ & $\begin{array}{c}\bar{X} \\
\text { Puntaje } \\
T \\
\end{array}$ & $\begin{array}{l}\text { Test } \\
\text { Sidak }\end{array}$ \\
\hline \multicolumn{10}{|l|}{ No Lector } \\
\hline Silábica & 3 & 49.3 & & 34.7 & & 25.7 & & 57.0 & \\
\hline Palabra a Palabra & 26 & 55.6 & $\mathrm{C}$ & 33.7 & $\mathrm{C}$ & 24.2 & $\mathrm{C}$ & 48.3 & B \\
\hline Unidades Cortas & 106 & 83.2 & B & 50.6 & B & 42.1 & B & 51.0 & B \\
\hline Fluida & 71 & 110.2 & A & 60.5 & A & 55.5 & A & 56.2 & A \\
\hline TOTAL & 206 & 88.5 & $<0.001$ & 51.6 & $<0.001$ & 44.2 & $<0.001$ & 52.5 & $<0.001$ \\
\hline
\end{tabular}

Tabla 16

Asociación entre Calidad de Lectura Oral y otras áreas de aprendizaje (4º́sico). Arauco, 2005

\begin{tabular}{lccccccc}
\hline \multirow{2}{*}{$\begin{array}{l}\text { CATEGORÍAS } \\
\text { LECTURA }\end{array}$} & $n$ & $\begin{array}{c}\text { VLO } \\
\mathrm{ppm}\end{array}$ & $\begin{array}{c}\text { Test } \\
\text { Sidak }\end{array}$ & $\begin{array}{c}\bar{X} \\
\text { \%Logro }\end{array}$ & $\begin{array}{c}\text { Test } \\
\text { Sidak }\end{array}$ & $\begin{array}{c}\text { Matemática } \\
\text { \%Logro }\end{array}$ & $\begin{array}{c}\text { Test } \\
\text { Sidak }\end{array}$ \\
\hline No Lector & & & & & & & \\
Silábica & 3 & 32.0 & $\mathrm{C}$ & 26.5 & $\mathrm{C}$ & 33.3 & $\mathrm{~B}$ \\
Palabra a Palabra & 12 & 46.3 & $\mathrm{C}$ & 46.6 & $\mathrm{BC}$ & 46.8 & $\mathrm{~B}$ \\
Unidades Cortas & 35 & 70.5 & $\mathrm{~B}$ & 55.1 & $\mathrm{~B}$ & 54.3 & $\mathrm{~B}$ \\
Fluida & 34 & 108.1 & $\mathrm{~A}$ & 72.7 & $\mathrm{~A}$ & 66.1 & $\mathrm{~A}$ \\
TOTAL & 84 & 80.9 & $<0.001$ & 60.0 & $<0.001$ & 57.3 & $<0.001$ \\
\hline
\end{tabular}

A modo de ejemplo, en la Tabla 14 se presentan los resultados de la muestra de 543 alumnos de Constitución 2004, por ser la más actual y en la que se analizaron varios cursos ${ }^{6}$.

Pudo observarse en este análisis una tendencia positiva en el promedio de velocidad de lectura de cada uno de los cursos conforme los alumnos presentaron un mayor nivel de calidad lectora, encontrándose diferencias significativas en el promedio de velocidad entre los niños que presentan distintas categorías de lectura, siendo los alumnos con Lectura Fluida los que presentaron una mayor velocidad lectora.

Mirada global de la asociación calidad de lectura-otras habilidades. Las Tablas 15 y 16 permiten dar una mirada simultánea a cómo evolucionan los resultados de los mismos alumnos en distintas habilidades, destrezas y manejo de contenidos de acuerdo al nivel de calidad de lectura oral alcanzado.

6 Los resultados obtenidos en las otras muestras estudiadas están disponibles en Fundar.
En la Tabla 15 se presentan los resultados que obtuvieron alumnos de $6^{\circ}$ Básico en velocidad de lectura, comprensión de lectura silenciosa, vocabulario y autoestima.

Puede observarse que los rendimientos obtenidos en $6^{\circ}$ básico tanto en velocidad lectora, vocabulario, comprensión lectora y autoestima mejoran conforme los alumnos logran mejores categorías de lectura. Los análisis realizados muestran diferencias significativas.

La Tabla 16 presenta los resultados que obtuvieron 91 alumnos de $4^{\circ}$ Básico en velocidad lectora y en manejo de contenidos en lenguaje y matemática, de acuerdo al nivel de calidad de lectura logrado. Los resultados de velocidad lectora aparecen expresados en palabras por minuto y los de manejo de contenidos en porcentaje de logro.

Puede observarse que los resultados alcanzados en $4^{\circ}$ Básico tanto en velocidad lectora, como en lenguaje y matemáticas mejoran conforme los alumnos leen mejor y que estas diferencias resultaron estadísticamente significativas. 
Estos resultados permiten dar cuenta de la asociación entre calidad de lectura oral y distintas habilidades. Corroboran que alumnos con mejor dominio lector rinden consistentemente mejor en destrezas fundamentales como la comprensión de lectura silenciosa y el manejo de vocabulario, rinden mejor en pruebas de contenidos tanto de lenguaje como de matemáticas y presentan una mejor autoestima de acuerdo a la percepción de los profesores.

\section{Conclusiones}

Los resultados presentados permiten reforzar las hipótesis planteadas, en cuanto a que existe asociación entre el nivel de calidad de lectura oral que presentan los alumnos de Educación Básica y el dominio de distintas habilidades como la comprensión de lectura silenciosa, el manejo de vocabulario y la redacción. También con el dominio de contenidos, no sólo del área de lenguaje sino de matemática y de comprensión del medio y con el desarrollo de la autoestima.

En las investigaciones hechas por Fundación Educacional Arauco en el tema asociación del Dominio Lector con otras habilidades, se utilizaron instrumentos válidos y confiables, estandarizados en Estados Unidos (Prueba de Lectura y Lenguaje Escrito PLLE), desarrollados y validados en nuestro país (Pruebas de Dominio Lector, TAE Profesor) o pruebas nacionales (SIMCE), lo que da gran respaldo a los resultados obtenidos.

Esta investigación avala la importancia del dominio lector como un elemento vital en todos los sectores de aprendizaje llegando a ser una destreza esencial en el desarrollo escolar y personal. La lectura no es un proceso que se adquiere como un todo. Es un proceso que se adquiere gradualmente en los primeros cursos de enseñanza básica y su aprendizaje se relaciona con la capacidad de dominar textos cada vez más complejos, captando su significado. Demuestra la importancia que el alumno domine y automatice la mecánica del proceso lo más tempranamente posible, y que no detenga su proceso en etapas iniciales o intermedias, para que pueda dirigir su atención a la comprensión y no a la decodificación. Muestra que el permanecer en etapas iniciales o intermedias afecta el desempeño de los alumnos en habilidades básicas, en manejo de contenidos y en el desarrollo de la autoestima.

Esta investigación nos señala que muchos alumnos que fueron parte de la muestra de este estudio no automatizan la lectura en los cursos de enseñan- za básica, no logran alcanzar los niveles mínimos esperados de lectura al inicio del año escolar, de acuerdo a los criterios de las pruebas. El dominio de la lectura debería constituirse, por lo tanto, en uno de los principales retos de toda escuela. El desafío es no sólo desarrollar el "gusto por leer" y enseñar la mecánica del proceso, sino hacerlo tempranamente ya que se comprueba que no da lo mismo aprender a leer bien en $2^{\circ}$ o en $5^{\circ}$ Básico ya que esa falta de dominio está afectando los resultados en otras áreas académicas y también en su desarrollo afectivo.

Los resultados nos muestran que no es lo mismo reconocer letras, leer sílabas o palabras, que leer fluidamente con una adecuada velocidad. Si un niño no es capaz de leer con suficiente calidad y rapidez, difícilmente será capaz de demostrar adecuadamente lo que sabe, por ejemplo, en una evaluación en que deba leer los textos e instrucciones en un tiempo delimitado. Un buen nivel lector permite al alumno centrar todos sus esfuerzos en la comprensión de los contenidos tratados y no en decodificar la información escrita a la que se ve enfrentado.

La investigación también refuerza la importancia de tener instrumentos en nuestro país que permitan evaluar lectura en forma objetiva, válida y confiable en los diferentes cursos de la enseñanza básica. El desempeño académico debe ser evaluado y retroalimentado en forma permanente. Las investigaciones en el ámbito de las escuelas efectivas demuestran que estos establecimientos registran minuciosamente los progresos de los alumnos para fines de planificación y de acción y que esto se hace tanto en habilidades como en contenidos. También hacen monitoreo a fin de enjuiciar el aprendizaje de los alumnos y comparan los resultados de las evaluaciones externas con los obtenidos en sala de clases (Brunner \& Elacqua, 2003; Teddlie \& Reynolds, 2000 citado en Marchant et al., 2004).

Resulta importante que en nuestro país existan pruebas válidas y confiables para evaluar lectura. Convendría seguir creando y validando instrumentos ya que éstos suelen perder validez cuando los profesores empiezan a usarlos como material de estimulación, cuando hay grandes avances en la investigación educativa o bien cuando el nivel general de los alumnos del país avanza y se quedan con estándares poco exigentes.

Esta investigación refuerza el concepto de que los profesores deberían estar capacitados en evaluar Dominio Lector a fin de formarse un criterio sobre las competencias en lectura que sus alumnos han alcanzado, individual y grupalmente, que además 
sirva de plataforma para reforzarlos y proyectar nuevas tareas. Con ello, al evaluar podrían apreciar las competencias parciales desarrolladas por los alumnos según las diversas etapas de aprendizaje de la lectura y detectar a los niños con necesidades educativas especiales en su aprendizaje lector. Esto les ayudaría no sólo a seleccionar adecuadas estrategias correctivas y remediales, sino a seleccionar y enriquecer el material de lectura de sus alumnos de acuerdo a sus competencias e intereses y a retroalimentar su propia eficacia.

Esta investigación también refuerza la importancia y el valor que tienen los instrumentos de screening o tamizaje. Estos son una evaluación gruesa que permite conocer el nivel de rendimiento en distintas habilidades en relación a una norma establecida y determinar si este rendimiento es adecuado o está bajo lo esperado. Las Pruebas de Dominio Lector Fundar, que son pruebas de screening o tamizaje en lectura, permiten al profesor conocer el grado de dominio lector de los alumnos con una metodología simple, de fácil aprendizaje. Los resultados les permiten a los profesores y especialistas no sólo saber como están los alumnos en esta importante destreza sino también proyectar lo que podría suceder con ellos en otras destrezas fundamentales como la redacción y el manejo de vocabulario, en su autoestima, en su manejo de contenidos, y también en sus resultados en evaluaciones externas como el SIMCE.

\section{Referencias}

Alliende, F. \& Condemarín, M. (1982). La lectura: Teoría, evaluación y desarrollo. Santiago: Editorial Andrés Bello.

Bravo, L. (2003). Lectura inicial y psicología cognitiva. Santiago: Ediciones Universidad Católica de Chile.

Bravo, L., Villalón, M. \& Orellana, E. (2002). La conciencia fonológica y la lectura inicial en niños que ingresan a primer año básico. Psykhe, 11, 175-182.

Brunner, J. J. \& Elacqua, G. (2003). Capital humano en Chile. Santiago: Percade Ediciones.

Connie, A. (2004). Lectura en voz alta. Recuperado el 13 de octubre de 2001, desde: http://elhogar.tripod.com/2002/200202/lectura_en_voz_alta.htm

Haeussler, I. M. \& Milicic, N. (1995). Confiar en uno mismo: Programa de autoestima. Santiago: Editorial Dolmen.

Hammill, D., Larsen, S., Wiederholt, J. \& Fountain-Chambers, J. (1982). PLLE: Prueba de Lectura y Lenguaje Escrito. Austin, TX: University of Texas.

Marchant, T., Haeussler, I. M. \& Torretti, A. (2002). TAE: Batería para evaluar autoestima escolar. Santiago: Ediciones Universidad Católica de Chile.

Marchant, T., Recart, I., Cuadrado, B. \& Sanhueza, J. (2004). Pruebas de dominio lector Fundar para alumnos de enseñanza básica. Santiago: Ediciones Universidad Católica de Chile.

Pardo, N. (2006). Insuficiencia en vocabulario, terapia del lenguaje y educación. Recuperado en octubre de 2006, desde http://espanol.geocities.com/taouniv/vocabulario.html

Paredes, G. \& Sassoon, Y. (2005). La lectura, la escritura y la autoestima. Recuperado en septiembre de 2006, desde http://www.correodelmaestro.com/anteriores/2005/noviembre/anteaula114.htm

UNESCO. (2005). Hacia las sociedades del conocimiento. Recuperado en septiembre de 2006, desde http://unesdoc.unesco. org/images/0014/001419/141908s.pdf. 